\title{
Neurological Bulletin
}

FEATURING ARTICLES BY TRAINEES IN NEUROLOGY \& NEUROSCIENCE

\section{Trigeminal Neuralgia Evolving Into Nummular Headache}

\author{
Bryan J. Cupka and Ivan S. Login \\ Department of Neurology, \\ University of Maryland, Baltimore, MD (BC) \\ Department of Neurology, \\ University of Virginia, Charlottesville, VA (IL)
}

\section{Introduction}

Nummular headache is a coin-shaped headache with varying degrees of severity. The pain is typically isolated to a small, rounded area of the head and is constant or intermittent in nature. Despite a growing amount of literature describing nummular headache, the exact etiology is unknown.

We report a case of an otherwise healthy 66year-old man who presented with an acute headache that was felt to be most consistent with trigeminal neuralgia (TN). With time, this headache evolved and developed characteristics that would fit the diagnostic criteria for nummular headache $(\mathrm{NH})$.

Despite speculation that NH could be related to pathology of the terminal branches of the trigeminal nerve, ${ }^{1}$ our literature review did not reveal cases of TN transforming into $\mathrm{NH}$. We feel this case adds important information to the growing literature about this headache syndrome.

\section{Case Report}

The patient is a healthy 66-year-old man with no previous headache history. He acutely developed a sharp, intermittent, retro-orbital pain, traveling into his left eye. There was no preceding head trauma, unusual physical exertion, seasonal allergies, or sinus disease. His right face was unaffected. The pain could also travel to the left occipital region. He denied any tearing or discharge from his left eye or his left ear, and he didn't report any loss of taste or smell. Naproxen and ibuprofen failed to offer relief. The headache rapidly worsened in severity and frequency over 12 days, and he was hospitalized at our institution overnight after an acute worsening during coitus.

At this time, his neurological examination was normal, including a completely normal cranial nerve examination. There was no cranio-cervical lymphadenopathy, head or neck mass, or sign of autonomic dysfunction. With concern for aneurysm, non-contrast head computed tomography (CT), magnetic resonance imaging (MRI) of the brain with-

Correspondence to Bryan Cupka: bcupka@som.umaryland.edu

Keywords: nummular, trigeminal, neuralgia 
out contrast, magnetic resonance angiogram (MRA) of the head and neck, erythrocyte sedimentation rate (ESR), and $\mathrm{C}$ reactive protein (CRP) were all normal. His vascular studies did not show any vascular loops or ectatic vessels. A complete blood count showed mild anemia, and a metabolic panel was normal except for a blood glucose of 113. The patient was observed overnight and received tramadol and intravenous ketorolac after no bleeding was found on imaging. Spinal fluid analysis was discussed but not performed due to rapid improvement in his pain. The next morning he had near complete resolution of his symptoms. The etiology remained unclear, although TN was discussed. Because of the improvement and lack of any headache history, we were reluctant to initiate preventive therapy. He was discharged with instructions to take over-thecounter analgesics as needed.

The headache returned several days after discharge with similar, but evolving, qualities. The pain had become more superficial, originating just anterior to his left ear. He described a mild to moderate constant pain, but also an intermittent, excruciating, lancinating pain lasting seconds to minutes toward his left eye and the left side of his face. There were no clear triggers, although activity may have made acute attacks more frequent. There was no jaw pain and no pain with chewing. There were no associated autonomic symptoms. This was now thought to be more consistent with $\mathrm{TN}$, and carbamazepine was started. This was titrated to $400 \mathrm{mg}$ twice daily with some improvement, but the pain was still present. Eventually, gabapentin $600 \mathrm{mg}$ nightly was added to carbamazepine $400 \mathrm{mg}$ twice daily, and he reported gradual, but highly effective improvement in the debilitating pain.

Around the same time, there was yet another striking change in the pain pattern. The orig- inal excruciating pain more consistent with $\mathrm{TN}$ disappeared, and now his headache was a $2 \mathrm{~cm}$ circular area located anterior and superior to his left ear. The pain was constantly present, and worsened by palpation. There had been one mild worsening of this new pain that lasted a few days, and then a return to baseline. His temporal artery was unremarkable on examination, and temporal arteritis was thought less likely given the normal ESR and CRP from prior. Neurological examination showed allodynia over the affected area, but was otherwise normal. Further review of his prior MRI revealed no underlying structural lesions. At this point he was given a diagnosis of $\mathrm{NH}$. A trial of nortriptyline 10 mg nightly improved the pain minimally, but was discontinued due to unacceptable side effects. A subsequent increase of gabapentin to $900 \mathrm{mg}$ nightly further lessened the severity of the pain. The carbamazepine was gradually discontinued, and the patient described stability in his symptoms at subsequent follow-up appointments.

Unfortunately, almost a year after onset, the intensity of the pain worsened over three weeks. It was acutely aggravated during a hair cut with frequent palpation of the affected area. The location was unchanged and there continued to be no other neurological symptoms. The gabapentin was increased from $900 \mathrm{mg}$ to $1200 \mathrm{mg}$ nightly as the patient was unable to tolerate any day time doses due to sedation. At his next appointment, he again described acute worsening of his symptoms after the affected area was palpated. At this time, his gabapentin dosing was divided to $300 \mathrm{mg}$ in the am, $300 \mathrm{mg}$ in the afternoon, and $600 \mathrm{mg}$ at night.

The patient is an otherwise quite healthy 66year-old with medical problems limited to hyperlipidemia, chronic lower back pain, cholecystectomy, depression, insomnia, and a history of diverticulitis. His pertinent med- 
Neurol. Bull. 4: 29-33, 2012

doi:10.7191/neurol_bull.2012.1036

ications at the onset of the headache included lamotrigine $150 \mathrm{mg}$ twice daily for depression, clonazepam $0.5 \mathrm{mg}$ daily for anxiety, and methadone $2.5 \mathrm{mg}$ four times daily as needed for back pain. The methadone is used infrequently, but most often after physical activity such as golf. Taking the methadone did not alter the headache characteristics, and the doses of lamotrigine and clonazepam were not changed during the course of the headache.

\section{Discussion}

We thought this case should be publicized because the clinical history and response to treatment support an initial diagnosis of $\mathrm{TN}$, seemingly evolving into NH. The International Headache Society (IHS) diagnostic criteria $^{2}$ for classical TN are:

A. Paroxysmal attacks of pain lasting from a fraction of a second to two minutes affecting one or more divisions of the trigeminal nerve and fulfilling criteria $\mathrm{B}$ and $\mathrm{C}$

B. Pain has at least one of the following characteristics:

1. Intense, sharp, superficial, or stabbing

2. Precipitated from trigger areas and trigger factors

C. Attacks are stereotyped in the individual patient

D. There is no clinically evident neurological deficit

E. Not attributed to another disorder

The original headaches fit these criteria. We recognize that the intermittent pain referred toward the left occipital region and the con- stant baseline pain between intermittent exacerbations early in this presentation would, however, be somewhat atypical for TN.

The IHS diagnostic criteria ${ }^{2}$ for $\mathrm{NH}$ are:

A. Mild to moderate head pain fulfilling criteria $\mathrm{B}$ and $\mathrm{C}$ :

B. Pain is felt exclusively in a rounded or elliptical area typically $2-6 \mathrm{~cm}$ in diameter

C. Pain is chronic and either continuous or interrupted by spontaneous remissions lasting weeks to months

\section{Not attributed to another disorder}

The more chronic portion of the headache fit these criteria. An extensive diagnostic work up did not find any other cause for the headache.

A review of the literature revealed descriptions of lancinating pain attacks with $\mathrm{NH}^{3,4}$ and a case of antecedent TN in a patient with $\mathrm{NH},{ }^{1}$ but no clear description of TN symptoms evolving into NH. Since the initial paper describing $\mathrm{NH},{ }^{4}$ many case series and reports have been published that have speculated on the possible etiology and pathophysiology of NH. There has been evidence presented favoring both peripheral and central etiologies, as well as descriptions of primary ${ }^{4}$ and secondary ${ }^{5} \mathrm{NH}$. Currently, it appears the majority of evidence supports a peripheral cause.

The effectiveness, or lack thereof, of specific treatments has also added valuable information to the literature. Neuropathic agents are frequently effective, such as gabapentin and carbamazepine in this case. Local anesthetics have been largely unsuccessful, ${ }^{4,6}$ but botulinum toxin type A injected directly into the scalp has been found to be helpful, possi- 
bly from direct antinociceptive properties. $^{7}$ A transcutaneous electrical nerve stimulation (TENS) unit was reportedly successful in another case report. ${ }^{8}$

Although trigeminal demyelination from vascular compression ${ }^{9,10}$ is a compelling theory for the etiology of clinical $\mathrm{TN}$, there are a variety of cellular, subcellular, neurotransmitter, and cytokine-mediated mechanisms that could also contribute secondarily to the severe pain. ${ }^{11}$ For example, ion channel dysfunction (potassium and sodium), neuropeptide modifications, and pro-inflammatory and pro-nociceptive cytokines ${ }^{11-13}$ are among additional mechanisms that may be relevant. If some or any of these do play a role in classical $\mathrm{TN}$, then it may be possible that these same mechanisms might also be relevant to NH. The concept of referred pain and changes in receptive field characteristics in the cranio-cervical region are also highly relevant, ${ }^{11}$ with the nucleus caudalis having a key role in redistributing the perception of pain from one cranial region to another. This, for example, might allow for some patients with $\mathrm{NH}$ to initially present with $\mathrm{TN}$, as we may have observed in our patient. Alternatively, there is evidence supporting the idea that analgesic use can cause a transformation from one headache syndrome to another, such as in medication-overuse headache and migraine. ${ }^{14}$ It could be considered that a similar type of pharmacologically induced transformation occurred in this case, although the medications we used are not widely considered to cause this phenomenon.

Given the uncertain pathophysiology of $\mathrm{NH}$, we feel this case adds valuable information to the growing literature about this disorder. We recognize that $\mathrm{NH}$ does not always localize to areas supplied by the trigeminal nerve, but for those that do, it may be important to investigate past episodes potentially consistent with TN. As NH can be difficult to treat medically at times, if a patient describes a history consistent with $\mathrm{TN}$, then a diagnostic evaluation focusing on the trigeminal nerve may be indicated. If the trigeminal nerve is found to be dysfunctional, interventional treatments classically used for $\mathrm{TN}$ could be discussed for medically refractory cases.

\section{References}

1. Pareja JA, Pareja J, Barriga FJ, et al. Nummular headache: a prospective series of 14 new cases. Headache 2004;44:611-614, http://dx.doi.org/10.1111/j.15264610.2004.446011.x

2. Headache Classification Committee of the International Headache Society. The International Classification of Headache Disorders, $2^{\text {nd }}$ edition. Cephalalgia 2004;24 Suppl. 1:126-127, 149, http:// dx.doi.org/10.1111/j.1468-

2982.2004.00653.x, http:// dx.doi.org/10.1111/j.14682982.2003.00828.x

3. Ruscheweyh R, Buchheister A, Gregor N, Jung A, Evers S. Nummular headache: six new cases and lancinating pain attacks as possible manifestation. Cephalalgia

2010;30:249-253, http:// dx.doi.org/10.1111/j.14682982.2009.01893.x

4. Pareja JA, Caminero AB, Serra J, et al. Nummular headache: a coin-shaped cephalgia. Neurology 2002;58:1678-1679, http://dx.doi.org/10.1212/ WNL.58.11.1678

5. Guillem A, Barriga FJ and GiménezRoldán S. Nummular headache secondary to an intracranial mass lesion. Cephalalgia 2007;27:943-944, http:// dx.doi.org/10.1111/j.14682982.2007.01328.x

6. Cohen GL. Nummular headache: what denomination? Headache 2005;45:1417- 
1418, http://dx.doi.org/10.1111/j.15264610.2005.00278 1.x

7. Mathew NT, Kailasam J and Meadors L. Botulinum toxin type A for the treatment of nummular headache: four case studies. Headache 2008;48:442-447, http:// dx.doi.org/10.1111/j.15264610.2007.00960.x

8. Tayeb Z, Hafeez F and Shafiq Q. Successful treatment of nummular headache with TENS. Cephalalgia 2008;28:897-898, http://dx.doi.org/10.1111/j.14682982.2007.01456.x

9. Devor M, Amir R and Rappaport ZH. Pathophysiology of trigeminal neuralgia: the ignition hypothesis. Clin J Pain 2002;18:4-13.

10. Love $S$ and Coakham HB. Trigeminal neuralgia: pathology and pathogenesis. Brain 2001;124:2347-2360, http:// dx.doi.org/10.1093/brain/124.12.2347

11. Iwata K, Imamura Y, Honda K, and Shinoda M. Physiological mechanisms of neuropathic pain: the orofacial region. Int Rev Neurobiol 2011;97:227-250, http:// dx.doi.org/10.1016/B978-0-12-3851987.00009-6

12. Takeda M, Tsuboi Y, Kitagawa J, Nakagawa $\mathrm{K}$, Iwata $\mathrm{K}$, and Matsumoto $\mathrm{S}$. Potassium channels as a potential therapeutic target for trigeminal neuropathic and inflammatory pain. Mol Pain 2011;7:5, http://dx.doi.org/10.1186/17448069-7-5

13. Sommer C, Kress M. Recent findings on how proinflammatory cytokines cause pain: peripheral mechanisms in inflammatory and neuropathic hyperalgesia. Neurosci Lett 2004;361:184-187, http:// dx.doi.org/10.1016/j.neulet.2003.12.007

14. Tepper S. Medication-overuse headache. Continuum 2012;18:807-822.
Disclosure: the authors report no conflicts of interest.

All content in Neurological Bulletin, unless otherwise noted, is licensed under a Creative Commons Attribution-Noncommercial-Share Alike License http://creativecommons.org/licenses/by-nc-sa/3.0/ (ISSN 1942-4043) 